\title{
Calibrating Accelerometers Using an Electromagnetic Launcher
}

\author{
E. J. Timpson P.E., T. G. Engel Ph. D. \\ Department of Electrical and Computer Engineering, University of Missouri, Columbia, MO, \\ etimpson@kcp.com
}

\begin{abstract}
A Pulse Forming Network (PFN), Helical Electromagnetic Launcher (HEML), Command Module (CM), and Calibration Table (CT) were built and evaluated for the combined ability to calibrate an accelerometer. The PFN has a maximum stored energy of $19.25 \mathrm{~kJ}$ bank and is fired by a silicon controlled rectifier (SCR), with appropriate safety precautions. The HEML is constructed out of $\mathbf{G - 1 0}$ fiberglass and is designed to accelerate 600 grams to 10 meters per second. The $C M$ is microcontroller based running Arduino Software. The CM has a keypad input and 7 segment outputs of the bank voltage and desired voltage. After entering a desired bank voltage, the $C M$ controls the charge of the PFN. When the two voltages are equal it allows the fire button to send a pulse to the SCR to fire the PFN and in turn, the HEML. The HEML projectile's tip hits a target that is held by the CT. The CT consists of a table to hold the PFN and HEML, a vacuum chuck, air bearing, velocity meter and catch pot. The Target is held with the vacuum chuck awaiting impact. After impact, the air bearing allows the target to fall freely for the velocity meter to get an accurate reading. A known acceleration is determined from the known change in velocity of the target. Thus, if an accelerometer was attached to the target, the measured value can be compared to the known value.
\end{abstract}

\section{INTRODUCTION AND BACKGROUND}

The US Department of Defense has considered electromagnetic launchers for weapons and other applications. In 2010, NASA released press that they were studying electric launchers for the first part of a journey to the stars. Helical launchers are the most promising of all types of electromagnetic launchers in terms of efficiency. This paper marks the first industry application of a HEML - the calibration of an accelerometer. Electromagnetic launchers are ideal for repeatability, controllability, high accelerations, velocities, and short travel requirements.

Accelerometers are calibrated with a number of processes. The process presented in this paper is common but with a different method for actuation - HEMLs. Other methods of actuation include elastics added gravity, pneumatics, and chemicals. All of which have some advantages, this work compares those advantages with electromagnetic launchers.

\section{A. Electromagnetic Launchers}

Over the years there has been considerable attention given to electromagnetic launchers. The major advantage of electromagnetic propulsion, at least for anti-armor mission, is the ability to reach higher impact velocities [1]. Higher velocities are important for many applications. For launching to Space with an electromagnetic gun Ian McNab said, "These techniques have the advantage that the launch mechanism remains on the Earth and does not have to be lifted into space, as with a rocket." [2] In the same paper he reviews gun options including electromagnetic rail guns, EM coilguns, electrothermal-chemical guns, light gas guns, RAM accelerators, blast wave accelerators, slingatron, and even lasers. In the end, because the government spent money and time on railguns, he chooses railguns. One-year later efficiency and scaling relationships for DC (i.e. noninduction) electromagnetic launchers opened a new door [3]. The exploration continued and formed a body of evidence [45]. Based on this evidence, the obvious choice is a HEML.

The HEML used in this paper operates in the same way as described in Reference 6. Reference 7 shows further progress in the art of making an HEML, specifically with the variable inductive gradient. The HEML in this paper does not require the variable inductive gradient as it operates at relative low velocities and energies in comparison.

\section{B. Calibration of Accelerometers}

There are two primary domains with regard to acceleration: magnitude and duration. The two are interrelated; however, calibration methods typically explore variations of each, independent of one another. Specifically, duration is evaluated with a shaker at many frequencies (e.g., $2 \mathrm{~Hz}$ to $10 \mathrm{kHz}$ ) and constant amplitude (e.g., $10 \mathrm{~g}$ ). We compared the test accelerometer to a Standard Accelerometer (Figure 1). The sensitivity derived from the vibration data helps in evaluating amplitude. Amplitude can be evaluated by using a reference accelerometer (i.e. Back to Back). Amplitude can also be evaluated by comparing with velocity change (i.e. the absolute method of calibration). The following is a step by step procedure of evaluating amplitude variation using the absolute method:

Step 1 - Attach accelerometer to the Target 


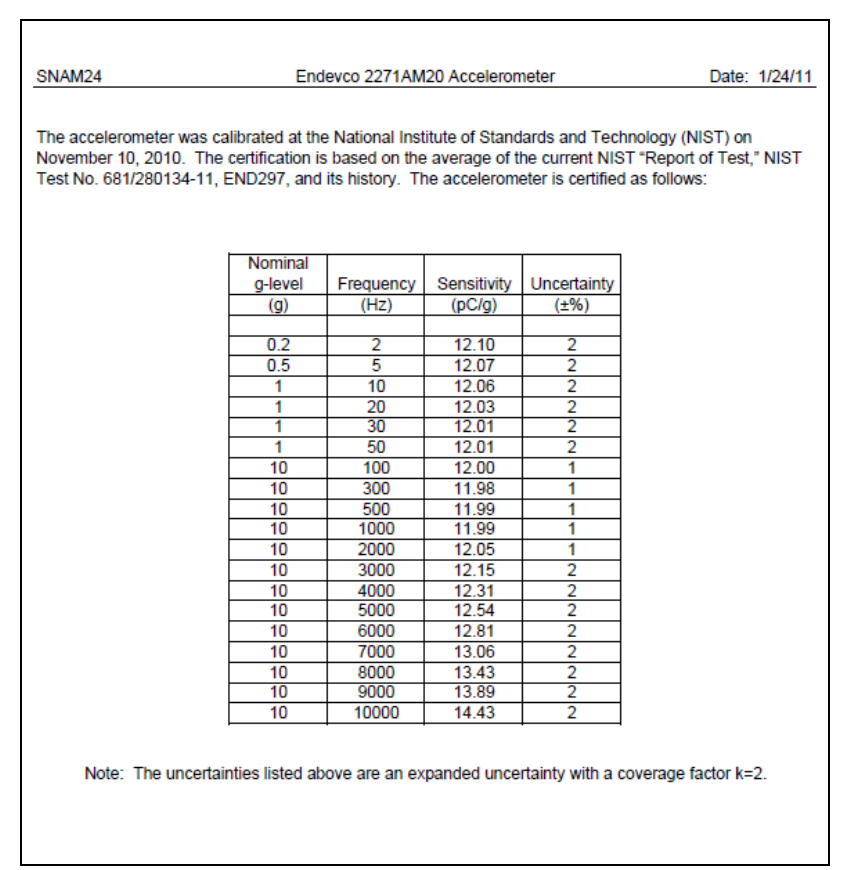

Figure 1. Standard Accelerometer Certification

Step 2 - Turn on Vacuum chuck and air bearing.

Step 3 - Place Target (with accelerometer attached) in the vacuum chuck air bearing assembly (Figure 2).

Step 4 - Ensure computer software, counter, and oscilloscope are ready to acquire data.

Step 5 - Deliver shock - Actuation

Step 6 - Analyze data. Compare accelerometer readout with velocity change information to acquire sensitivity.

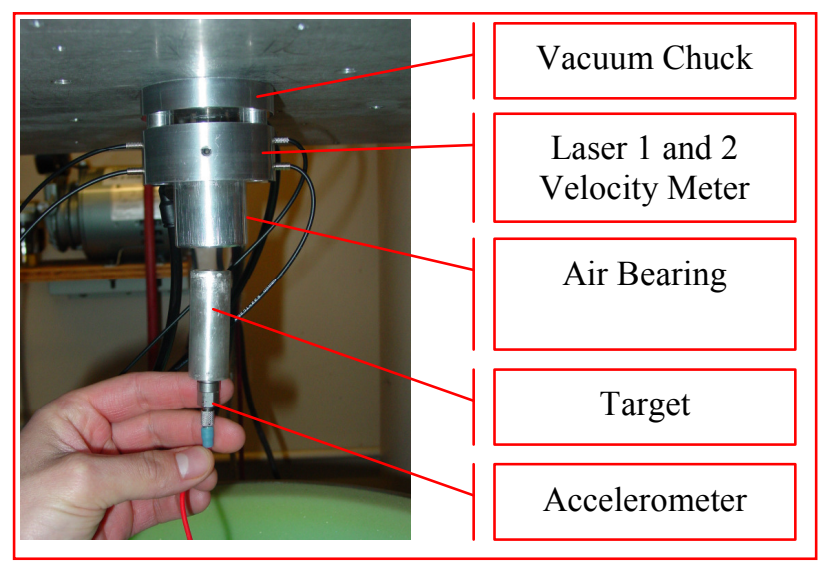

Figure 2. Accelerometer Calibration Setup Below the Table.

Traditionally, actuation takes place by bungee cord and bow release.

\section{System DesigN}

A new system was developed using an electromagnetic launcher as the actuator. The Calibration Table (CT) and in turn the calibration uncertainty is based off of the well know methods discussed in the introduction. The CT consists of a table to hold the Pulse Forming Network (PFN) and HEML. Attached to the bottom of the CT are a vacuum chuck, air bearing, and laser velocity meter. Below the Calibration Table is a catch pot (Figure 3 - green foam filled pot).

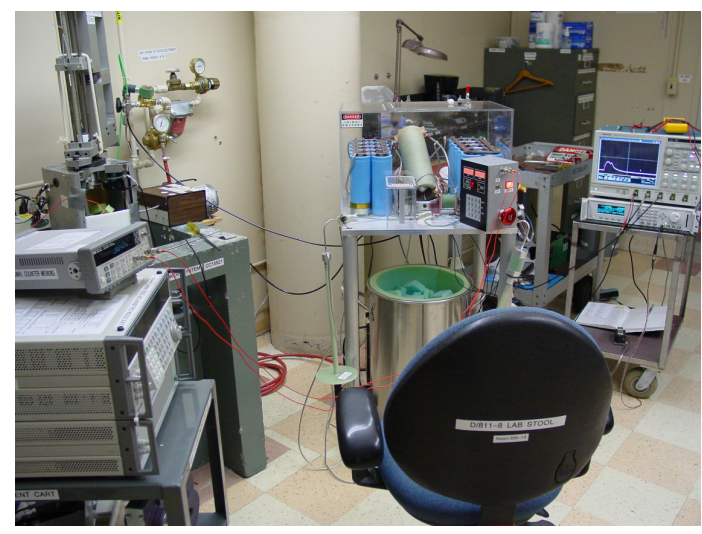

Figure 3. Entire system

The Command Module (CM) was built new for this application. In an effort to get the same pull-it-back-and-letit-go operation, simplicity was the driving factor. An Arduino microcontroller is the heart of the Proof of Concept CM. The microcontroller communicates with the user by seven segment displays (and other LEDs). The user communicates with the microcontroller with a keypad and Fire button. One can see the CM in Figure 3 as the black box on the CT. The CM's job is to monitor and control the PFN. This includes: 1) Monitor safety switches (including an emergency stop) for human exposure to High voltage and immediately discharge the capacitors if energized. 2) Await the desired bank Voltage from the user. 3) Upon obtaining confirmation from the user, the $\mathrm{CM}$ charges the Capacitor Bank to the Desired Voltage (with a High Voltage Power supply) 4) The CM stops charging at the desired voltage. 5) The CM awaits user input to fire the HEML and upon receiving this input discharges the capacitors into the HEML.

As mentioned, Reference 6 contains a detailed description of the HEML, its operating principles and equations. In short, the HEML is comprised of a barrel and projectile. One can see the Barrel in Figure 3 (a green tube at a slant to expose the shiny projectile tip). The Barrel is essentially a coil of wire with two insolated rails for providing current to the projectile. The projectile (Figure 4) is essentially another coil of wire with contacts to the rails and barrel coil. Current flows from one rail through the two coils of wires (barrel and projectile) to the other rail. Large current through the two coils creates two large opposing magnetic fields. The opposition accelerates the projectile, allowing the Tool 
Hardened steel tip to contact the Target through a hole in the CT.

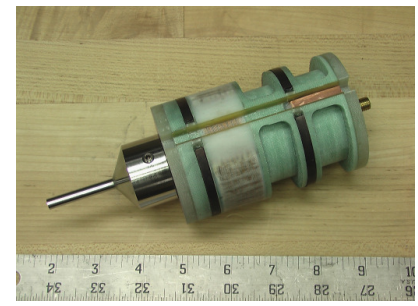

Figure 4. HEML projectile with Tool Hardened tip

\section{A. Calibration and Experiment}

Figure 5 illustrates the sequence of events. Figure 6 describes the variables in Figure 5.

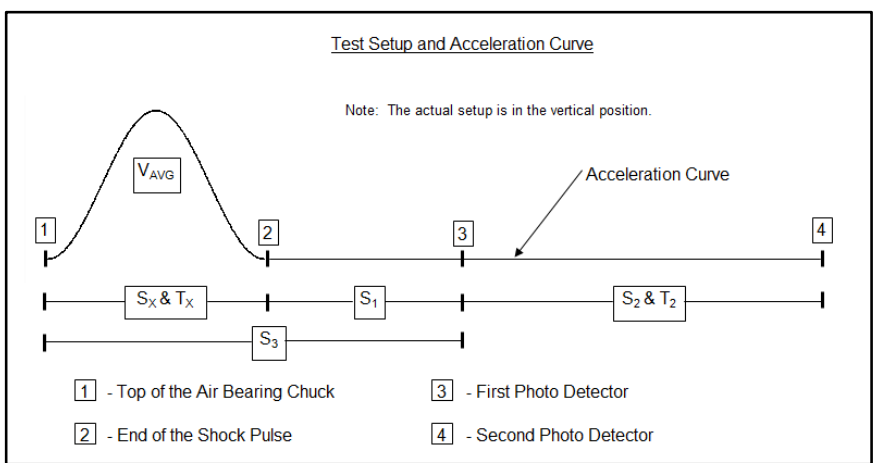

Figure 5. Test Setup and Acceleration Curve

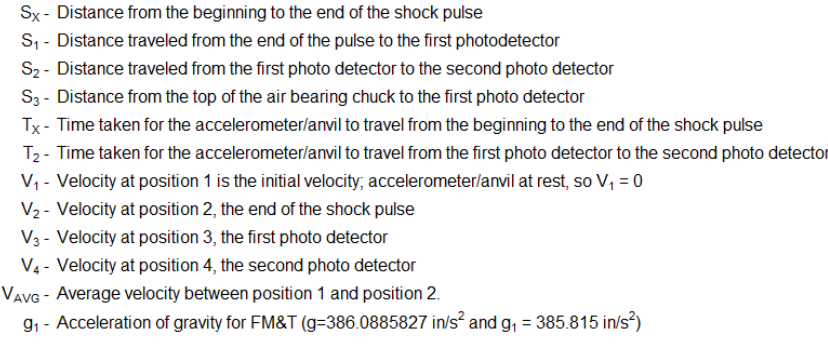

Figure 6. Variable explanation

Figure 7 illustrates a collection of equations that are derived from basic Physics and Figure 5.

\begin{tabular}{llll}
\hline & Equations & \\
(1) $V_{4}=V_{3}+g_{1} \times T_{2}$ & "(3) $V_{3}^{2}=V_{2}^{2}+2 \times g_{1} \times S_{1}$ & (5) $S_{X}=T_{X} \times V_{A V G}$ \\
(2) $V_{4}^{2}=V_{3}^{2}+2 \times g_{1} \times S_{2}$ & "(4) $V_{A V G}=\frac{\left(V_{1}+V_{2}\right)}{2}$ & (6) $S_{3}=S_{1}+S_{X}$
\end{tabular}

Figure 7. Equation Collection

The calibration parameter is sensitivity (e.g., $0.1 \mathrm{mV}$ per g). The sensitivity is calculated using Equation 7 .

$$
\mathrm{SC}=\left(\mathrm{g}_{1} \times \mathrm{A} \times \mathrm{SF}\right) / \mathrm{V}_{2}
$$

Gravity $\left(\mathrm{g}_{1}\right)$ is in Figure 6. Area under the shock pulse (A) is taken off the oscilloscope integrating function (It also equals $\mathrm{V}_{\mathrm{s}} * \mathrm{~T}_{\mathrm{s}}$, or pulse width times the amplitude). The signal conditioning Scale Factor (SF) is one because the accelerometer under test used a signal conditioner (vs a capacitor or charge amplifier). Velocity change $\left(\mathrm{V}_{2}\right)$ is the change in velocity from position 1 to position 2 . The velocity at position 1 is 0 , so the velocity change is the velocity at position 2. $\mathrm{S}_{2}$ and $\mathrm{T}_{2}$ are measured quantities so starting with equation (1) or (2) will lead us to the calculation of $V_{2}$. Here we use equation (1). Let $\mathrm{V}_{4}=\mathrm{ds} / \mathrm{dt}$, solve for $\mathrm{ds}$ and integrate:

$$
\begin{gathered}
\frac{d s}{d t}=V_{3}+g_{1} \times T_{2} \\
d s=\left(V_{3}+g_{1} \times T_{2}\right) \times d t \\
\int_{0}^{S_{2}} d s=\int_{0}^{T_{2}}\left(V_{3}+g_{1} \times T_{2}\right) d t \\
S_{2}=V_{3} \times T_{2}+\frac{1}{2} \times g_{1} \times T_{2}^{2}
\end{gathered}
$$

Solve for $\mathrm{V}_{3}$

$$
V_{3}=\frac{S_{2}}{T_{2}}-\frac{1}{2} \times g_{1} \times T_{2}
$$

Substitute Equation (12) into Equation (3) and solve for $\mathrm{V}_{2}$ :

$$
V_{2}=\sqrt{\left(\frac{S_{2}}{T_{2}}-\frac{1}{2} \times g_{1} \times T_{2}\right)^{2}-2 \times g_{1} \times S_{1}}
$$

Note: Equation (13) is in Reference [10] on page 146. Solve Equation (4) for $\mathrm{V}_{\mathrm{AVG}}$ when $\mathrm{V}_{1}$ is zero.

$$
V_{A V G}=\frac{V_{2}}{2}
$$

Substitute $\mathrm{V}_{\mathrm{AVG}}$ into Equation (5)

$$
S_{X}=\frac{1}{2} \times T_{X} \times V_{2}
$$

Solve Equation (6) for $S_{x}$, substitute into Equation (9), and solve for $\mathrm{S}_{1}$ 


$$
S_{1}=S_{3}-\frac{1}{2} \times T_{X} \times V_{2}
$$
$\mathrm{V}_{2}$.

Substitute Equation (10) into Equation (8) and solve for

$V_{2}^{2}+\left(-g_{1} \times T_{X}\right) \times V_{2}+\left[2 \times g_{1} \times S_{3}-\left(\frac{S_{2}}{T_{2}}-\frac{1}{2} \times g_{1} \times T_{2}\right)^{2}\right]=0$

Using the quadratic formula and that the negative solution is less than zero and $V_{2}$ cannot be less than zero, the $V_{2}$ solution is as follows:

$V_{2}=\frac{1}{2} \times g_{1} \times T_{X}+\sqrt{\frac{S_{2}^{2}}{T_{2}^{2}}-2 \times g_{1} \times S_{3}-g_{1} \times S_{2}+\frac{1}{4} \times g_{1}^{2} \times T_{X}^{2}+\frac{1}{4} \times g_{1}^{2} \times T_{2}^{2}}$

Equation (18) is in the calibration software. The calibration software also talks to the counter and oscilloscope using GPIB.

The experiment was to calibrate the same accelerometer using the bungee cord and, bow release method, and compare that to the new Electromagnetic Launcher Method.

\section{B. Uncertainty Evaluation}

We used the NIST GUM method to construct the Uncertainty Analysis [11]. We shall start by defining all uncertainties in Figure 8.

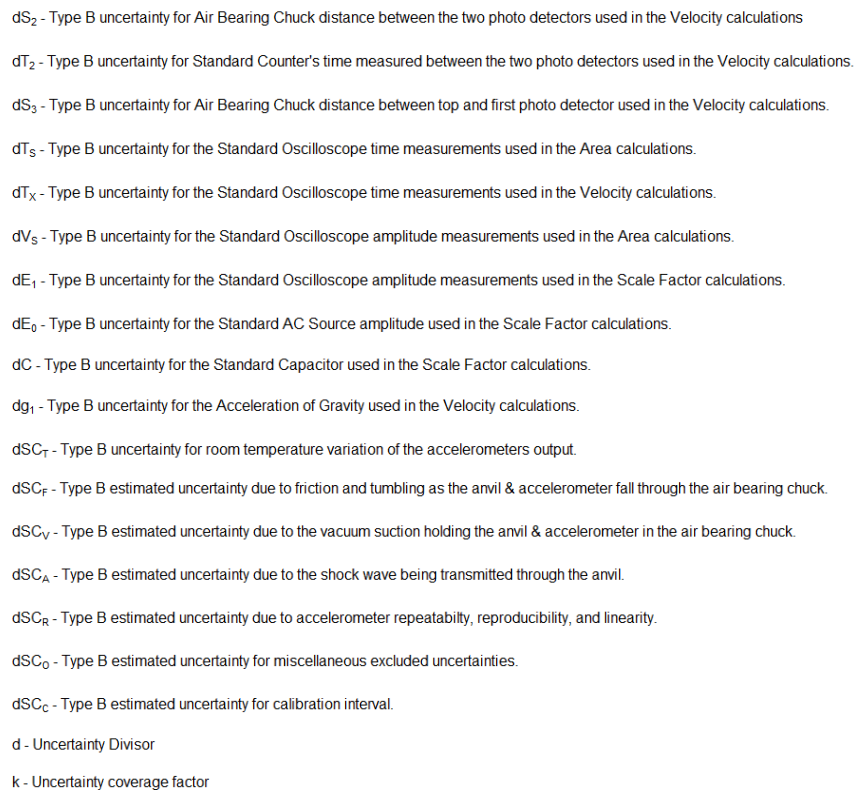

Figure 8. Uncertainty Definitions
After defining all uncertainties, one must use partial differentials to determine the magnitude each has on the overall system. Equation (19) is the system equation (7) including the mathematical progress thus far.

$$
S C=\frac{g_{1} \times V_{S} \times T_{S} \times 1}{\frac{1}{2} \times g_{1} \times T_{X}+\sqrt{\frac{S_{2}^{2}}{T_{2}^{2}}-2 \times g_{1} \times S_{3}-g_{1} \times S_{2}+\frac{1}{4} \times g_{1}^{2} \times T_{X}^{2}+\frac{1}{4} \times g_{1}^{2} \times T_{2}^{2}}}
$$

In the interest of simplifying the differentials, new terms represent the numerator and the components of the denominator.

$$
N_{1}=g_{1} \times V_{S} \times T_{S}
$$

$$
D_{1}=\frac{1}{2} \times g_{1} \times T_{X}
$$

$$
D_{2}=\sqrt{\frac{S_{2}^{2}}{T_{2}^{2}}-2 \times g_{1} \times S_{3}-g_{1} \times S_{2}+\frac{1}{4} \times g_{1}^{2} \times T_{X}^{2}+\frac{1}{4} \times g_{1}^{2} \times T_{2}^{2}}
$$

$$
S C=\frac{N_{1}}{D_{1}+D_{2}}
$$

Now the Partial differentials follow:

$$
\frac{\partial N_{1}}{\partial g_{1}}=V_{S} \times T_{S}=\frac{N_{1}}{g_{1}}
$$

$$
\begin{aligned}
& \frac{\partial N_{1}}{\partial V_{S}}=g_{1} \times T_{S}=\frac{N_{1}}{V_{S}} \\
& \frac{\partial N_{1}}{\partial T_{S}}=g_{1} \times V_{S}=\frac{N_{1}}{T_{S}}
\end{aligned}
$$

$$
\frac{\partial D_{1}}{\partial g_{1}}=\frac{1}{2} \times T_{X}=\frac{D_{1}}{g_{1}}
$$

$$
\frac{\partial D_{1}}{\partial T_{X}}=\frac{1}{2} \times g_{1}=\frac{D_{1}}{T_{X}}
$$

$$
\frac{\partial D_{2}}{\partial S_{2}}=\frac{2 \times S_{2}-g_{1} \times T_{2}^{2}}{2 \times D_{2} \times T_{2}^{2}}
$$

$$
\frac{\partial D_{2}}{\partial S_{3}}=\frac{-g_{1}}{D_{2}}
$$

$$
\frac{\partial D_{2}}{\partial T_{X}}=\frac{g_{1}^{2} \times T_{X}}{4 \times D_{2}}
$$




$$
\begin{gathered}
\frac{\partial D_{2}}{\partial T_{2}}=\frac{-4 \times S_{2}^{2}+g_{1}^{2} \times T_{2}^{4}}{4 \times D_{2} \times T_{2}^{3}} \\
\frac{\partial D_{2}}{\partial g_{1}}=\frac{-4 \times S_{3}-2 \times S_{2}+g_{1} \times T_{X}^{2}+g_{1} \times T_{2}^{2}}{4 \times D_{2}} \\
\frac{\partial S C}{\partial N_{1}}=\frac{1}{D_{1}+D_{2}}=\frac{S C}{N_{1}} \\
\frac{\partial S C}{\partial D_{1}}=\frac{-N_{1}}{\left(D_{1}+D_{2}\right)^{2}} \\
\frac{\partial S C}{\partial D_{2}}=\frac{-N_{1}}{\left(D_{1}+D_{2}\right)^{2}}
\end{gathered}
$$

Now we can combine using the RSS method.

$$
\begin{gathered}
d N_{1}=\sqrt{\left(\frac{\partial N_{1}}{\partial g_{1}} \times d g_{1}\right)^{2}+\left(\frac{\partial N_{1}}{\partial V_{S}} \times d V_{S}\right)^{2}+\left(\frac{\partial N_{1}}{\partial T_{S}} \times d T_{S}\right)^{2}} \\
d D_{1}=\sqrt{\left(\frac{\partial D_{1}}{\partial g_{1}} \times d g_{1}\right)^{2}+\left(\frac{\partial D_{1}}{\partial T_{X}} \times d T_{X}\right)^{2}} \\
d S C=\sqrt{\left(\frac{\partial D_{2}}{\partial S_{2}} \times d S_{2}\right)^{2}+\left(\frac{\partial D_{2}}{\partial T_{2}} \times d T_{2}\right)^{2}+\left(\frac{\partial D_{2}}{\partial g_{1}} \times d g_{1}\right)^{2}+\left(\frac{\partial D_{2}}{\partial S_{3}} \times d S_{3}\right)^{2}+\left(\frac{\partial D_{2}}{\partial T_{X}} \times d T_{X}\right)^{2}} \\
\left.d S N_{1}\right)^{2}+\left(\frac{\partial S C}{\partial D_{1}} \times d D_{1}\right)^{2}+\left(\frac{\partial S C}{\partial D_{2}} \times d D_{2}\right)^{2}
\end{gathered}
$$

\section{RESULTS}

\section{A. Calibration Results}

Using the calibration procedure explained in Section I and II we calibrated an accelerometer using the old method and the new method. The accelerometer was a Kistler 8044 using a signal conditioner. The same sensitivity was determined using both methods. The sensitivity was $0.1016 \mathrm{mV} / \mathrm{g} \pm$ $2.13 \%$. Figure 9 shows the shock pulse obtained with the new system. The amplitude was $11,432 \mathrm{~g}$ with a duration of $0.120 \mathrm{~ms}$. The maximum bungee cord acceleration was $18,000 \mathrm{~g}$ at a pulse width of $0.100 \mathrm{~ms}$. The highest acceleration recorded on the HEML system was $22,441 \mathrm{~g}$ at a pulse width of $0.1 \mathrm{~ms}$. This was only operating at 400 volts or $3.08 \mathrm{~kJ}$ (not even $10 \%$ of the maximum capacity). The bungee cord method requires 42 centimeters of travel distance before hitting that target to get an acceleration of $18 \mathrm{~kg}$. The HEML operates at $2.54 \mathrm{~cm}$ independent of acceleration.

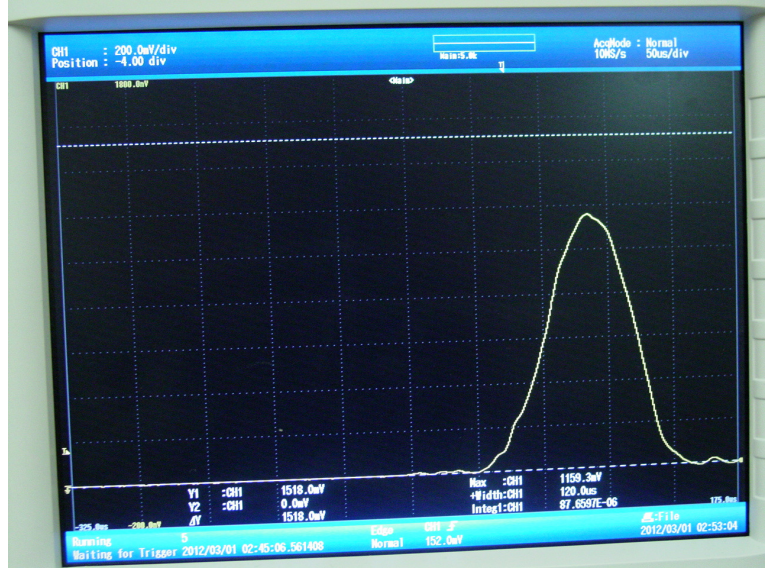

Figure 9. Calibration shock pulse

\section{B. Comparison to other methods}

The bungee cord method does have advantages. The largest advantage is simplicity; and because of that, timesaving (pull-it-back-and-let-it-go). Disadvantages to a bungee cord and bow release are operator dependence, large tower requirement for large amplitudes, and limitation on maximum acceleration. The HEML system has clear advantages over the bungee cord method in that it has no operator dependence; there is a considerable travel distance reduction, and no theoretical limit on velocity. The clear downside to the HEML launcher, because no PFN optimization has been done is the increased time to operate.

Pneumatics also has advantages; in fact, the most popular actuation for High Amplitude and frequency shock pulses is an Air gun [8-9]. Air guns maximum velocity is limited by the speed of sound, whereas an Electromagnetic launcher (EML) is not. It would be a wonderful future work to see a Hopkinson Bar operated with an electromagnetic Launcher. Further one could research from an energy storage perspective the cost of using an electric generator to fill up a tank (pneumatics) vs. the cost of directly charging the capacitors. In the case of power tools, especially ones used infrequently there is a clear energy savings of electrics over pneumatics.

Chemicals propellants have some advantages also. This work did not explore what specifically they would be; however, the clear downside to chemicals in comparison to electromagnetic launchers is the ease of repeatability with less cost in expendable materials.

\section{CONCLUSIONS}

An Electromagnetic Launcher given the right setup can calibrate an accelerometer. The HEML setup resulted in the same sensitivity as the bungee cord method. The HEML setup exceeded the maximum acceleration of the old system operating at $16 \%$ of the maximum energy. This work also suggests the exploration of Electromagnetic Launchers in a wide range of industry applications - specifically the calibration of accelerometers. 


\section{ACKNOWLEDGMENT}

Gratitude is extended toward Matthew Clewell and Randy Herder for providing valuable contributions in many aspects of this work.

\section{REFERENCES}

[1] Scanlon, J.J., III; Batteh, J.H.; Chryssomallis, G.; , "Tactical applications for electromagnetic launchers," Magnetics, IEEE Transactions on , vol.31, no.1, pp.552-557, Jan 1995 doi:

URL:

$10.1109 / 20.364634$

http://ieeexplore.ieee.org/stamp/stamp.jsp?tp=\&arnumber=364634\&isn umber $=8355$

[2] McNab, I.R.; , "Launch to space with an electromagnetic railgun," Magnetics, IEEE Transactions on, vol.39, no.1, pp. 295- 304, Jan 2003 doi:

10.1109/TMAG.2002.805923

URL:

http://ieeexplore.ieee.org/stamp/stamp.jsp?tp=\&arnumber=1179826\&is number $=26497$

[3] Engel, T.G.; Nunnally, W.C.; Gahl, J.M.; , "Efficiency and Scaling in DC Electromagnetic Launchers," Pulsed Power Conference, 2005 IEEE ， vol., no., pp.249-252, 13-17 June 2005 doi: $\quad 10.1109 /$ PPC.2005.300589

URL:

http://ieeexplore.ieee.org.proxy.mul.missouri.edu/stamp/stamp.jsp?tp= \&arnumber $=4084199 \&$ isnumber $=4084141$

[4] Engel, T.G.; Neri, J.M.; Nunnally, W.C.; , "A Same-Scale Comparison of Electromagnetic Launchers," Power Modulator Symposium, 2006. Conference Record of the 2006 Twenty-Seventh International , vol., no., $\quad$ pp.405-410, 14-18 May 2006 doi: $\quad$ 10.1109/MODSYM.2006.365270

URL:

http://ieeexplore.ieee.org.proxy.mul.missouri.edu/stamp/stamp.jsp?tp= \&arnumber $=4216222 \&$ isnumber $=4216116$

[5] Engel, T.G.; Veracka, M.J.; Neri, J.M.; , "The Specific-Force Performance Parameter for Electromagnetic Launchers," Plasma Science, IEEE Transactions on , vol.38, no.2, pp.194-198, Feb. 2010 doi:

URL:

10.1109/TPS.2009.2036260

http://ieeexplore.ieee.org.proxy.mul.missouri.edu/stamp/stamp.jsp?tp= \&arnumber $=5342526 \&$ isnumber $=5410033$

[6] Engel, T.G.; Neri, J.M.; Veracka, M.J.; , "Solid-Projectile Helical Electromagnetic Launcher," Plasma Science, IEEE Transactions on, vol.37, no.4, pp.603-607, April 2009 doi: $\quad$ 10.1109/TPS.2009.2012714

http://ieeexplore.ieee.org.proxy.mul.missouri.edu/stamp/stamp.jsp?tp= \&arnumber $=4776443 \&$ isnumber $=4812340$

[7] Engel, T.G.; Veracka, M.J.; , "Solid-Projectile Helical Electromagnetic Launcher With Variable Gradient Stator and Magnetically Levitated Armature," Plasma Science, IEEE Transactions on , vol.39, no.12, pp.3371-3377, Dec. 2011 doi:

10.1109/TPS.2011.2168570

URL:

http://ieeexplore.ieee.org.proxy.mul.missouri.edu/stamp/stamp.jsp?tp= \&arnumber $=6036183 \&$ isnumber $=6086645$

[8] Bateman, V.I.; Leisher, W.B.; Brown, F.A.; Davie, N.T., "Calibration of a Hopkinson bar with a transfer standard," Presented at the $62^{\text {nd }}$ Shock and Vibration Symposium, Springfield, VA, Oct. 1991

[9] Bateman, V.I.; Brown F.A.; Davie N.T., "Use of a Beryllium Hopkinson Bar To Characterize a Piezoresistive Accelerometer In Shock Environments," Journal of the Institute of Environmental Sciences, vol. 39, no. Nov. 1996

[10] Bouche, R.R., "Calibration of Shock and Vibration Measuring Transducers," Navel Research Laboratory, 1979, pp. 12 \& 144-151, 1979

[11] Kuyatt, C.E.; Taylor, B.N., "Guide for Evaluating and Expressing the uncertainty of NIST Measuremet Results" NIST Technical Note 1297 1994
This work has been funded by Honeywell Federal Manufacturing \& Technologies under Contract No.DE-NA-0000622 with the U.S. Department of Energy. The United States Government retains and the publisher, by accepting the article for publication, acknowledges that the United states Government retains a nonexclusive, paid-up, irrevocable, world-wide license to publish or reproduce the published form of this manuscript, or allow others to do so, for United States Government purposes. 